\title{
On class A operators
}

by

Sungeun Jung, Eungil Ko and MeE-Jung LeE (Seoul)

\begin{abstract}
We show that every class A operator has a scalar extension. In particular, such operators with rich spectra have nontrivial invariant subspaces. Also we give some spectral properties of the scalar extension of a class A operator. Finally, we show that every class A operator is nonhypertransitive.
\end{abstract}

1. Introduction. Let $\mathcal{H}$ be a complex separable Hilbert space and let $\mathcal{L}(\mathcal{H})$ denote the algebra of all bounded linear operators on $\mathcal{H}$. If $T \in$ $\mathcal{L}(\mathcal{H})$, we write $\sigma(T), \sigma_{\text {ap }}(T)$, and $\sigma_{\mathrm{e}}(T)$ for the spectrum, the approximate point spectrum, and the essential spectrum, respectively, and write $r(T)=\sup \{|\lambda|: \lambda \in \sigma(T)\}$ for the spectral radius of $T$. An operator $T \in$ $\mathcal{L}(\mathcal{H})$ is said to be $p$-hyponormal if $\left(T T^{*}\right)^{p} \leq\left(T^{*} T\right)^{p}$, where $0<p<\infty$. In particular, 1-hyponormal operators and $\frac{1}{2}$-hyponormal operators are called hyponormal operators and semi-hyponormal operators, respectively.

An arbitrary operator $T \in \mathcal{L}(\mathcal{H})$ has a unique polar decomposition $T=$ $U|T|$, where $|T|=\left(T^{*} T\right)^{1 / 2}$ and $U$ is a partial isometry satisfying $\operatorname{ker} U=$ $\operatorname{ker}|T|=\operatorname{ker} T$ and $\operatorname{ker} U^{*}=\operatorname{ker} T^{*}$. Associated with $T$ is the operator $|T|^{1 / 2} U|T|^{1 / 2}$ called the Aluthge transform of $T$, and denoted throughout this paper by $\widehat{T}$. For every $T \in \mathcal{L}(\mathcal{H})$, the sequence $\left\{\widehat{T}^{(n)}\right\}$ of Aluthge iterates of $T$ is defined by $\widehat{T}^{(0)}=T$ and $\widehat{T}^{(n+1)}=\widehat{T}^{(n)}$ for every positive integer $n$ (see [2], [15], and [16]).

An operator $T \in \mathcal{L}(\mathcal{H})$ is said to be w-hyponormal if $|\widehat{T}| \geq|T| \geq\left|\widehat{T}^{*}\right|$ (see [3]), and paranormal if $\|T x\|^{2} \leq\left\|T^{2} x\right\|\|x\|$ for all $x \in \mathcal{H}$. We say that $T \in \mathcal{L}(\mathcal{H})$ is normaloid if $\|T\|=r(T)$. It is well-known that every $p$-hyponormal operator is $w$-hyponormal and that every $w$-hyponormal operator is normaloid. Furuta-Ito-Yamazaki ([12]) introduced the following interesting class of Hilbert space operators.

2010 Mathematics Subject Classification: Primary 47B20; Secondary 47A11.

Key words and phrases: class A operator, scalar extension, invariant subspace, nonhypertransitive. 
Definition 1.1. An operator $T \in \mathcal{L}(\mathcal{H})$ is said to belong to class $A$ if it satisfies the condition $\left|T^{2}\right| \geq|T|^{2}$.

It is known from [12] that

\{hyponormal operators $\} \subset\{p$-hyponormal operators $\}(0<p \leq 1)$

$$
\begin{aligned}
& \subset\{\text { class A operators }\} \\
& \subset\{\text { paranormal operators }\} \\
& \subset\{\text { normaloid operators }\} .
\end{aligned}
$$

There is a vast literature concerning class A operators ([1]-14], 27], 28], etc.). By a simple computation one can show that a weighted shift belongs to class A if and only if it is hyponormal. In [11], T. Furuta gives several examples of class A operators, including the following.

EXAmple $1.2([11])$. Let $A=\left(\begin{array}{cc}17 & 7 \\ 7 & 5\end{array}\right)^{2}$ and $B=\left(\begin{array}{ll}1 & 0 \\ 0 & 4\end{array}\right)^{2}$ be operators on $\mathbb{R}^{2}$, and let $\mathcal{H}_{n}=\mathbb{R}^{2}$ for all positive integers $n$. Consider the operator $T_{A, B}$ on $\bigoplus_{n=1}^{\infty} \mathcal{H}_{n}$ defined by

$$
T_{A, B}=\left(\begin{array}{ccccccc}
\ddots & \vdots & \vdots & \vdots & \vdots & \vdots & \\
\cdots & B & 0 & 0 & 0 & 0 & \cdots \\
\cdots & 0 & B & \widehat{0} & 0 & 0 & \cdots \\
\cdots & 0 & 0 & B & 0 & 0 & \cdots \\
\cdots & 0 & 0 & 0 & A & 0 & \cdots \\
\cdots & 0 & 0 & 0 & 0 & A & \cdots \\
& \vdots & \vdots & \vdots & \vdots & \vdots & \ddots
\end{array}\right)
$$

where the hat indicates the position of the $(0,0)$ element in the matrix. Then $T_{A, B}$ is a class A operator, but is not $p$-hyponormal for any $p$.

An operator $S \in \mathcal{L}(\mathcal{H})$ is called scalar of order $m$ if it possesses a spectral distribution of order $m$, i.e. a continuous unital morphism of topological algebras

$$
u: C_{0}^{m}(\mathbb{C}) \rightarrow \mathcal{L}(\mathcal{H})
$$

such that $u(z)=S$, where as usual $z$ stands for the identity function on $C_{0}^{m}$, the complex-valued continuously differentiable functions of order $m$, $0 \leq m \leq \infty$. An operator is said to be subscalar of order $m$ if it is similar to the restriction of a scalar operator of order $m$ to an invariant subspace.

In 1984, M. Putinar [24] showed that every hyponormal operator has a scalar extension. In 1987, his theorem was used to show that hyponormal operators with thick spectra have nontrivial invariant subspaces, a result due to S. Brown (see [7]). In this paper we generalize those theorems to the context of class A operators. In fact, we show that every class A operator is 
subscalar of order 12. In particular, every class A operator whose spectrum has nonempty interior has a nontrivial invariant subspace. Also we give some spectral properties of the scalar extension of a class A operator. Finally, we consider the hypertransitive operator problem, i.e., the question whether $(\mathrm{NHT})=\mathcal{L}(\mathcal{H})$ (defined later). In particular, we show that every class A operator is nonhypertransitive.

2. Preliminaries. An operator $T \in \mathcal{L}(\mathcal{H})$ is said to have the singlevalued extension property at $z_{0}$ if for every neighborhood $D$ of $z_{0}$ and any analytic function $f: D \rightarrow \mathcal{H}$ with $(T-z) f(z) \equiv 0$, we have $f(z) \equiv 0$. An operator $T \in \mathcal{L}(\mathcal{H})$ is said to have the single-valued extension property (or $\mathrm{SVEP}$ ) if it has the single-valued extension property at every $z$ in $\mathbb{C}$. For an operator $T \in \mathcal{L}(\mathcal{H})$ with SVEP and for $x \in \mathcal{H}$ we can consider the set $\rho_{T}(x)$ of elements $z_{0}$ in $\mathbb{C}$ such that there exists an analytic function $f(z)$ defined in a neighborhood of $z_{0}$, with values in $\mathcal{H}$, which satisfies $(T-z) f(z) \equiv x$. We denote $\sigma_{T}(x)=\mathbb{C} \backslash \rho_{T}(x)$ and $\mathcal{H}_{T}(F)=\left\{x \in \mathcal{H}: \sigma_{T}(x) \subset F\right\}$, where $F$ is a subset of $\mathbb{C}$. An operator $T \in \mathcal{L}(\mathcal{H})$ is said to have Dunford's property $(\mathrm{C})$ if $\mathcal{H}_{T}(F)$ is closed for each closed subset $F$ of $\mathbb{C}$. An operator $T \in \mathcal{L}(\mathcal{H})$ is said to have the property $(\beta)$ if for every open subset $G$ of $\mathbb{C}$ and every sequence $f_{n}: G \rightarrow \mathcal{H}$ of $\mathcal{H}$-valued analytic functions such that $(T-z) f_{n}(z)$ converges uniformly to 0 in norm on compact subsets of $G, f_{n}(z)$ converges uniformly to 0 in norm on compact subsets of $G$. It is well-known that

$$
\text { Property }(\beta) \Rightarrow \text { Dunford's property }(C) \Rightarrow \text { SVEP. }
$$

An operator $T \in \mathcal{L}(\mathcal{H})$ with SVEP is said to have the decomposition property $(\delta)$ (or simply the property $(\delta)$ ) if $\mathcal{H}=\mathcal{H}_{T}(\bar{U})+\mathcal{H}_{T}(\bar{V})$ for every open cover $\{U, V\}$ of $\mathbb{C}$. It is well-known that the adjoint of a bounded linear operator on a Hilbert space with the property $(\beta)$ has the property $(\delta)$ (see [1]).

Let $z$ be the coordinate in $\mathbb{C}$, and let $d \mu(z)$, or simply $d \mu$, denote the planar Lebesgue measure. Let $U$ be a bounded open subset of $\mathbb{C}$. We shall denote by $L^{2}(U, \mathcal{H})$ the Hilbert space of measurable functions $f: U \rightarrow \mathcal{H}$ such that

$$
\|f\|_{2, U}=\left(\int_{U}\|f(z)\|^{2} d \mu(z)\right)^{1 / 2}<\infty .
$$

We denote the space $L^{2}(U, \mathcal{H}) \cap H(U, \mathcal{H})$ by $A^{2}(U, \mathcal{H})$, where $H(U, \mathcal{H})$ is the Fréchet space of analytic (holomorphic) $\mathcal{H}$-valued functions on $U$. Then $A^{2}(U, \mathcal{H})$ is a closed subspace of the $L^{2}(U, \mathcal{H})$, and the orthogonal projection of $L^{2}(U, \mathcal{H})$ onto this space will be denoted by $P$.

Now, we introduce a special Sobolev type space. Let $U$ be a bounded open subset of $\mathbb{C}$ and $m$ be a fixed nonnegative integer. Then the Sobolev space $W^{m}(U, \mathcal{H})$ is the space of functions $f \in L^{2}(U, \mathcal{H})$ whose derivatives 
$\bar{\partial} f, \bar{\partial}^{2} f, \ldots, \bar{\partial}^{m} f$ in the sense of distributions still belong to $L^{2}(U, \mathcal{H})$. Endowed with the norm

$$
\|f\|_{W^{m}}^{2}=\sum_{i=0}^{m}\left\|\bar{\partial}^{i} f\right\|_{2, U}^{2},
$$

$W^{m}(U, \mathcal{H})$ becomes a Hilbert space contained continuously in $L^{2}(U, \mathcal{H})$. The linear operator $M$ of multiplication by $z$ on $W^{m}(U, \mathcal{H})$ is continuous and it has a spectral distribution $u$ of order $m$ defined by the following relation: for $\varphi \in C_{0}^{m}(\mathbb{C})$ and $f \in W^{m}(U, \mathcal{H}), u(\varphi) f=\varphi f$. Hence $M$ is a scalar operator of order $m$.

3. Main results. In this section, we show that every class A operator has a scalar extension. For this, we begin with the following lemma which is the key step to prove our main theorem.

Lemma 3.1. Let $T \in \mathcal{L}(\mathcal{H})$ be a class $A$ operator and let $D$ be any bounded disk containing $\sigma(T)$. Define the map $V: \mathcal{H} \rightarrow H(D)$ by

$$
V h=\widetilde{1 \otimes h}\left(\equiv 1 \otimes h+\overline{(T-z) W^{12}(D, \mathcal{H})}\right),
$$

where $H(D)=W^{12}(D, \mathcal{H}) / \overline{(T-z) W^{12}(D, \mathcal{H})}$ and $1 \otimes h$ denotes the constant function sending $z \in D$ to $h$. Then $V$ is one-to-one and has closed range.

Proof. Let $h_{n} \in \mathcal{H}$ and $f_{n} \in W^{12}(D, \mathcal{H})$ be sequences which satisfy

$$
\lim _{n \rightarrow \infty}\left\|(T-z) f_{n}+1 \otimes h_{n}\right\|_{W^{12}}=0 .
$$

Then by the definition of the norm of the Sobolev space, (3.1) implies that

$$
\lim _{n \rightarrow \infty}\left\|(T-z) \bar{\partial}^{i} f_{n}\right\|_{2, D}=0
$$

for $i=1, \ldots, 12$. From $(3.2)$ we get

$$
\lim _{n \rightarrow \infty}\left\|\left(T^{2}-z^{2}\right) \bar{\partial}^{i} f_{n}\right\|_{2, D}=0
$$

for $i=1, \ldots, 12$. Let $T^{2}=U_{2}\left|T^{2}\right|$ and $\widehat{T^{2}}=V\left|\widehat{T^{2}}\right|$ be the polar decompositions of $T^{2}$ and $\widehat{T^{2}}$, respectively. Since $\widehat{T^{2}}\left|T^{2}\right|^{1 / 2}=\left|T^{2}\right|^{1 / 2} T^{2}$ and $\left.{\widehat{T^{2}}}^{(2)}\left|{\widehat{T^{2}}}^{1 / 2}=\right| \widehat{T}^{2}\right|^{1 / 2} \widehat{T^{2}}$, we have

$$
\left\{\begin{array}{l}
\lim _{n \rightarrow \infty}\left\|\left(\widehat{T^{2}}-z^{2}\right) \bar{\partial}^{i}\left|T^{2}\right|^{1 / 2} f_{n}\right\|_{2, D}=0, \\
\lim _{n \rightarrow \infty}\left\|\left(\widehat{T}^{(2)}-z^{2}\right) \bar{\partial}^{i}\left|\widehat{T^{2}}\right|^{1 / 2}\left|T^{2}\right|^{1 / 2} f_{n}\right\|_{2, D}=0,
\end{array}\right.
$$

for $i=1, \ldots, 12$. Since $T$ belongs to class A, from [13, $T^{2}$ is a $w$-hyponormal operator, and so $\widehat{T^{2}}$ is semi-hyponormal and ${\widehat{T^{2}}}^{(2)}$ is hyponormal by the definition of a $w$-hyponormal operator and [3]. Hence, it follows from (3.4) 
that

$$
\lim _{n \rightarrow \infty}\left\|\left(\widehat{T}^{2}(2)-z^{2}\right)^{*} \bar{\partial}^{i}\left|\widehat{T}^{2}\right|^{1 / 2}\left|T^{2}\right|^{1 / 2} f_{n}\right\|_{2, D}=0
$$

for $i=1, \ldots, 12$. By Theorem 3.1 of [18], there exists a constant $C_{D}$ such that

$$
\begin{aligned}
\|(I-P) \bar{\partial}^{i}\left|\widehat{T}^{2}\right|^{1 / 2} & \left|T^{2}\right|^{1 / 2} f_{n} \|_{2, D} \\
& \leq C_{D} \sum_{j=2+i}^{4+i}\left\|\left(\widehat{T}^{2}(2)-z^{2}\right)^{*} \bar{\partial}^{j}\left|\widehat{T^{2}}\right|^{1 / 2}\left|T^{2}\right|^{1 / 2} f_{n}\right\|_{2, D}
\end{aligned}
$$

for $i=0,1, \ldots, 8$, where $P$ denotes the orthogonal projection of $L^{2}(D, \mathcal{H})$ onto the Bergman space $A^{2}(D, \mathcal{H})$. From 3.5 and $(3.6)$, we obtain

$$
\lim _{n \rightarrow \infty}\left\|(I-P) \bar{\partial}^{i}\left|\widehat{T^{2}}\right|^{1 / 2}\left|T^{2}\right|^{1 / 2} f_{n}\right\|_{2, D}=0
$$

for $i=1, \ldots, 8$. Thus, by 3.4 and 3.7,

$$
\lim _{n \rightarrow \infty}\left\|\left(\widehat{T}^{2}(2)-z^{2}\right) P \bar{\partial}^{i}\left|\widehat{T}^{2}\right|^{1 / 2}\left|T^{2}\right|^{1 / 2} f_{n}\right\|_{2, D}=0
$$

for $i=1, \ldots, 8$. Since ${\widehat{T^{2}}}^{(2)}$ is hyponormal, it has the property $(\beta)$. Hence

$$
\lim _{n \rightarrow \infty}\left\|P \bar{\partial}^{i}\left|\widehat{T^{2}}\right|^{1 / 2}\left|T^{2}\right|^{1 / 2} f_{n}\right\|_{2, D_{0}}=0
$$

for $i=1, \ldots, 8$, where $\sigma(T) \subsetneq D_{0} \subsetneq D$. From 3.7 and 3.9 , we get

$$
\lim _{n \rightarrow \infty}\left\|\left|\widehat{T^{2}}\right|^{1 / 2}\left|T^{2}\right|^{1 / 2} \bar{\partial}^{i} f_{n}\right\|_{2, D_{0}}=0
$$

for $i=1, \ldots, 8$. Since $\widehat{T^{2}}\left|T^{2}\right|^{1 / 2}=\left|T^{2}\right|^{1 / 2} T^{2}$, from 3.3 and 3.10 we obtain

$$
\lim _{n \rightarrow \infty}\left\|z^{4} \bar{\partial}^{i} f_{n}\right\|_{2, D_{0}}=0
$$

for $i=1, \ldots, 8$. By Theorem 3.1 of [18], there exists a constant $C_{D_{0}}$ such that

$$
\left\|(I-P) f_{n}\right\|_{2, D_{0}} \leq C_{D_{0}} \sum_{i=4}^{8}\left\|z^{4} \bar{\partial}^{i} f_{n}\right\|_{2, D_{0}} .
$$

By (3.11) and (3.12), it follows that

$$
\lim _{n \rightarrow \infty}\left\|(I-P) f_{n}\right\|_{2, D_{0}}=0 .
$$

Combining (3.13) with 3.1 , we have

$$
\lim _{n \rightarrow \infty}\left\|(T-z) P f_{n}+1 \otimes h_{n}\right\|_{2, D_{0}}=0 .
$$

Let $\Gamma$ be a curve in $D_{0}$ surrounding $\sigma(T)$. Then

$$
\lim _{n \rightarrow \infty}\left\|P f_{n}(z)+(T-z)^{-1}\left(1 \otimes h_{n}\right)(z)\right\|=0
$$


uniformly for all $z \in \Gamma$. Applying the Riesz-Dunford functional calculus, we obtain

$$
\lim _{n \rightarrow \infty}\left\|\frac{1}{2 \pi i} \int_{\Gamma} P f_{n}(z) d z+h_{n}\right\|=0 .
$$

But by Cauchy's theorem, $\frac{1}{2 \pi i} \int_{\Gamma} P f_{n}(z) d z=0$. Hence

$$
\lim _{n \rightarrow \infty}\left\|h_{n}\right\|=0 \text {. }
$$

So, $V$ is one-to-one and has closed range.

Now we are ready to show that every class A operator has a scalar extension.

Theorem 3.2. Every class A operator in $\mathcal{L}(\mathcal{H})$ is subscalar of order 12.

Proof. Let $T \in \mathcal{L}(\mathcal{H})$ be a class A operator, let $D$ be an arbitrary bounded open disk in $\mathbb{C}$ that contains $\sigma(T)$ and consider the quotient space

$$
H(D)=W^{12}(D, \mathcal{H}) / \overline{(T-z) W^{12}(D, \mathcal{H})}
$$

endowed with the Hilbert space norm. The class of a vector $f$ or an operator $S$ on $H(D)$ will be denoted by $\widetilde{f}$, respectively $\widetilde{S}$. Let $M$ be multiplication by $z$ on $W^{12}(D, \mathcal{H})$. As noted at the end of Section $2, M$ is a scalar operator of order 12 and has a spectral distribution $u$. Since the range of $T-z$ is invariant under $M, \widetilde{M}$ is well-defined. Moreover, consider the spectral distribution $u$ : $C_{0}^{12}(\mathbb{C}) \rightarrow \mathcal{L}\left(W^{12}(D, \mathcal{H})\right)$ defined by the following relation: for $\varphi \in C_{0}^{12}(\mathbb{C})$ and $f \in W^{12}(D, \mathcal{H}), u(\varphi) f=\varphi f$. Then the spectral distribution $u$ of $M$ commutes with $T-z$, and so $\widetilde{M}$ is still a scalar operator of order 12 with $\widetilde{u}$ as a spectral distribution. Consider the operator $V: \mathcal{H} \rightarrow H(D)$ given by $V h=\widetilde{1 \otimes h}$ and denote the range of $V$ by $\operatorname{ran} V$. Since

$$
V T h=\widetilde{1 \otimes T h}=\widetilde{z \otimes h}=\widetilde{M}(\widetilde{1 \otimes h})=\widetilde{M} V h
$$

for all $h \in \mathcal{H}$, we have $V T=\widetilde{M} V$. In particular, $\operatorname{ran} V$ is invariant under $\widetilde{M}$. Furthermore, it is closed by Lemma 3.1, and hence it is a closed invariant subspace of the scalar operator $\widetilde{M}$. Since $T$ is similar to the restriction $\left.\widetilde{M}\right|_{\text {ranV }}$, and $\widetilde{M}$ is a scalar operator of order $12, T$ is subscalar of order 12 .

Theorem 3.2 has the following corollary.

Corollary 3.3 .

(i) Every p-hyponormal or w-hyponormal operator is subscalar.

(ii) If $T \in \mathcal{L}(\mathcal{H})$ is a class $A$ operator, then $f(T)$ is subscalar for every function $f$ analytic on a neighborhood of $\sigma(T)$.

Proof. (i) Since every $p$-hyponormal and every $w$-hyponormal operator belongs to class A by Section 1, the assertion follows from Theorem 3.2 . 
(ii) Let $T$ be a class A operator and let $f$ be an analytic function on a neighborhood of $\sigma(T)$. With the same notations as in the proof of Theorem 3.2, we have $V f(T)=f(\widetilde{M}) V$. Thus $f(T)$ is subscalar.

Recall from [6] that an operator $T \in \mathcal{L}(\mathcal{H})$ is said to be power regular if $\lim _{n \rightarrow \infty}\left\|T^{n} h\right\|^{1 / n}$ exists for every $h \in \mathcal{H}$.

Corollary 3.4.

(i) Every class A operator satisfies the property $(\beta)$, Dunford's property $(\mathrm{C})$, and the single-valued extension property.

(ii) Every class A operator is power regular.

Proof. (i) Let $T \in \mathcal{L}(\mathcal{H})$ be a class A operator. It suffices to prove that $T$ has the property $(\beta)$. Since the property $(\beta)$ is transmitted from an operator to its restrictions to closed invariant subspaces, we are reduced by Theorem 3.2 to the case of a scalar operator of order 12 . Since every scalar operator has the property $(\beta)$ (see [24]), $T$ has the property $(\beta)$.

(ii) Let $T \in \mathcal{L}(\mathcal{H})$ be a class A operator. Since $T$ is subscalar of order 12 from Theorem 3.2 , it is the restriction of a scalar operator of order 12 to one of its closed invariant subspaces. Since a scalar operator is power regular and all restrictions of power regular operators to their invariant subspaces clearly remain power regular, $T$ is power regular.

Recall that an operator $X \in \mathcal{L}(\mathcal{H}, \mathcal{K})$ is called a quasiaffinity if it has trivial kernel and dense range. An operator $S \in \mathcal{L}(\mathcal{H})$ is said to be a quasiaffine transform of $T \in \mathcal{L}(\mathcal{K})$ if there is a quasiaffinity $X \in \mathcal{L}(\mathcal{H}, \mathcal{K})$ such that $X S=T X$. Furthermore, $S$ and $T$ are quasisimilar if there are quasiaffinities $X$ and $Y$ such that $X S=T X$ and $S Y=Y T$.

Corollary 3.5. Let $C$ and $D$ in $\mathcal{L}(\mathcal{H})$ belong to class $A$. If $C$ and $D$ are quasisimilar, then $\sigma(C)=\sigma(D)$ and $\sigma_{\mathrm{e}}(C)=\sigma_{\mathrm{e}}(D)$.

Proof. Since $C$ and $D$ satisfy the property $(\beta)$ from Corollary 3.4 , the assertion follows from [25].

Next we will give some applications of Theorem 3.2 including a partial solution of the invariant subspace problem for class A operators. Moreover, the following theorem is a generalization of S. Brown's theorem and Berger's theorem (see [7] and [5]).

Theorem 3.6. Let $T \in \mathcal{L}(\mathcal{H})$ be a class $A$ operator.

(i) If $\sigma(T)$ has nonempty interior in $\mathbb{C}$, then $T$ has a nontrivial invariant subspace.

(ii) There exists a positive integer $K$ such that for all positive integers $k \geq K, T^{2 k}$ has a nontrivial invariant subspace. 
Proof. (i) This follows from Theorem 3.2 and 9 .

(ii) From [13, $T^{2}$ is a $w$-hyponormal operator. Therefore, by [5] there exists a positive integer $K$ such that for all positive integers $k \geq K, T^{2 k}$ has a nontrivial invariant subspace.

Next we study some spectral properties of the scalar extension of a class A operator.

TheOREM 3.7. Let $T \in \mathcal{L}(\mathcal{H})$ be a class A operator. With the notation of the proof of Theorem 3.2 , $\sigma_{\widetilde{M}}(V h)=\sigma_{T}(h)$ for each $h \in \mathcal{H}$.

Proof. Let $h \in \mathcal{H}$. If $\lambda_{0} \in \rho_{T}(h)$, then there is an $\mathcal{H}$-valued analytic function $g$ defined on a neighborhood $U$ of $\lambda_{0}$ such that $(T-\lambda) g(\lambda)=h$ for all $\lambda \in U$. Then

$$
(\widetilde{M}-\lambda) V g(\lambda)=V(T-\lambda) g(\lambda)=V h
$$

for all $\lambda \in U$. Hence $\lambda_{0} \in \rho_{\widetilde{M}}(V h)$. That is, $\rho_{\widetilde{M}}(V h) \supset \rho_{T}(h)$.

Conversely, suppose $\lambda_{0} \in \rho_{\widetilde{M}}(V h)$. Then there exists an $H(D)$-valued analytic function $\widetilde{f}$ on some neighborhood $U$ of $\lambda_{0}$ such that $(\widetilde{M}-\lambda) \widetilde{f}(\lambda)=$ $V h$ for all $\lambda \in U$. Let $f \in H\left(U, W^{12}(D, \mathcal{H})\right)$ be a holomorphic lifting of $\widetilde{f}$ and fix $\zeta \in U$. Then $h-(\zeta-z) f(\zeta, z) \in \overline{(T-z) W^{12}(D, \mathcal{H})}$. Therefore, there is a sequence $\left\{g_{n}\right\} \subset H\left(U, W^{12}(D, \mathcal{H})\right)$ such that

$$
\lim _{n \rightarrow \infty}\left\|h-(\zeta-z) f(\zeta, z)-(T-z) g_{n}(\zeta, z)\right\|_{W^{12}}=0
$$

with respect to $z \in U$. Then

$$
\lim _{n \rightarrow \infty}\left\|h-(T-z) f_{n}\right\|_{W^{12}}=0
$$

where $f_{n}(z):=g_{n}(z, z)$ for $z \in U$. From the proof of Lemma 3.1 (cf. (3.13)), we obtain

$$
\lim _{n \rightarrow \infty}\left\|(I-P) f_{n}\right\|_{2, U_{0}}=0
$$

where $U_{0}$ is an open neighborhood of $\lambda_{0}$ with $U_{0} \subsetneq U$, and so

$$
\lim _{n \rightarrow \infty}\left\|h-(T-z) P f_{n}\right\|_{2, U_{0}}=0 .
$$

This implies $h \in \overline{(T-z) H\left(U_{0}, \mathcal{H}\right)}$. Since $T$ has the property $(\beta)$ from Corollary 3.4, the operator $T-z$ has closed range on $H\left(U_{0}, \mathcal{H}\right)$. Thus $h \in$ $(T-z) H\left(U_{0}, \mathcal{H}\right)$, i.e., $\lambda_{0} \in \rho_{T}(h)$.

Corollary 3.8. Let $T \in \mathcal{L}(\mathcal{H})$ be a class $A$ operator. With the notation of the proof of Theorem $3.2, \sigma(T)=\sigma(\widetilde{M})$.

Proof. Since $\sigma_{T}(h)=\sigma_{\widetilde{M}}(V h)$ for all $h \in \mathcal{H}$ by Theorem 3.7. $\sigma_{T}(h) \subset$ $\sigma(\widetilde{M})$ for all $h \in \mathcal{H}$. Hence $\bigcup\left\{\sigma_{T}(h): h \in \mathcal{H}\right\} \subset \sigma(\widetilde{M})$. Since $T$ has the single valued extension property by Corollary 3.4, it follows that $\sigma(T)=$ $\bigcup\left\{\sigma_{T}(h): h \in \mathcal{H}\right\} \subset \sigma(\widetilde{M})$. 
Conversely, note that if $U \subset \mathbb{C}$ is any open disk containing $\sigma(T)$ and $M$ is multiplication by $z$ on $W^{12}(U, \mathcal{H})$, then $\sigma(\widetilde{M}) \subset \sigma(M) \subset \bar{U}$. From this property, if $\lambda \in \rho(T)$, then we can choose an open disk $D$ so that $\widetilde{M}-\lambda$ is invertible. Since this algebraic property is independent of the choice of $D$, we get $\sigma(\widetilde{M}) \subset \sigma(T)$.

Recall that a closed subspace of $\mathcal{H}$ is said to be hyperinvariant for $T$ if it is invariant under every operator in the commutant $\{T\}^{\prime}$ of $T$. An operator $T \in \mathcal{L}(\mathcal{H})$ is decomposable provided that, for each open cover $\{U, V\}$ of $\mathbb{C}$, there exist closed $T$-invariant subspaces $Y, Z$ of $\mathcal{H}$ such that $\mathcal{H}=Y+Z$, $\sigma\left(\left.T\right|_{Y}\right) \subset U$, and $\sigma\left(\left.T\right|_{Z}\right) \subset V$. Here, $\left.T\right|_{Y}$ denotes the restriction of $T$ to $Y$.

Theorem 3.9. Let $T \in \mathcal{L}(\mathcal{H})$ be a class $A$ operator and let $T \neq z I$ for all $z \in \mathbb{C}$. If $S$ is a decomposable quasiaffine transform of $T$ or $\lim _{n \rightarrow \infty}\left\|T^{n} h\right\|^{1 / n}$ $<\|T\|$ for some nonzero $h \in \mathcal{H}$, then $T$ has a nontrivial hyperinvariant subspace.

Proof. If $S$ is a decomposable quasiaffine transform of $T$, then there exists a quasiaffinity $X$ such that $X S=T X$ where $S$ is decomposable. If $T$ has no nontrivial hyperinvariant subspace, we may assume that $\sigma_{\mathrm{p}}(T)=\emptyset$ and $\mathcal{H}_{T}(F)=\{0\}$ for each closed set $F$ proper in $\sigma(T)$ by Lemma 3.6.1 of [19. Let $\{U, V\}$ be an open cover of $\mathbb{C}$ with $\sigma(T) \backslash \bar{U} \neq \emptyset$ and $\sigma(T) \backslash \bar{V} \neq \emptyset$. If $x \in \mathcal{H}_{S}(\bar{U})$, then $\sigma_{S}(x) \subset \bar{U}$. So there exists an analytic $\mathcal{H}$-valued function $f$ defined on $\mathbb{C} \backslash \bar{U}$ such that $(S-z) f(z) \equiv x$ for all $z \in \mathbb{C} \backslash \bar{U}$. Hence $(T-z) X f(z)=X(S-z) f(z)=X x$ for all $z \in \mathbb{C} \backslash \bar{U}$. Thus $\mathbb{C} \backslash \bar{U} \subset$ $\rho_{T}(X x)$, which implies that $X x \in \mathcal{H}_{T}(\bar{U})$, i.e., $X \mathcal{H}_{S}(\bar{U}) \subset \mathcal{H}_{T}(\bar{U})$. Similarly, $X \mathcal{H}_{S}(\bar{V}) \subset \mathcal{H}_{T}(\bar{V})$. Then since $S$ is decomposable,

$$
X \mathcal{H}=X \mathcal{H}_{S}(\bar{U})+X \mathcal{H}_{S}(\bar{V}) \subseteq \mathcal{H}_{T}(\bar{U})+\mathcal{H}_{T}(\bar{V})=\{0\} .
$$

But this is a contradiction. So $T$ has a nontrivial hyperinvariant subspace.

Now suppose that $\lim _{n \rightarrow \infty}\left\|T^{n} h\right\|^{1 / n}<\|T\|$ for some nonzero $h \in \mathcal{H}$. Since $T$ is a class A operator,

$$
\|T x\|^{2}=\left\langle|T|^{2} x, x\right\rangle \leq\left\langle\left|T^{2}\right| x, x\right\rangle \leq\left\|\left|T^{2}\right| x\right\|\|x\| \leq\left\|T^{2} x\right\|\|x\|
$$

for every $x \in \mathcal{H}$. This implies that

$$
\left\|T^{n} x\right\|^{2}=\left\|T T^{n-1} x\right\|^{2} \leq\left\|T^{2} T^{n-1} x\right\|\left\|T^{n-1} x\right\|=\left\|T^{n+1} x\right\|\left\|T^{n-1} x\right\|
$$

for every positive integer $n$ and every $x \in \mathcal{H}$. Hence, Proposition 4.6 and a remark in [6] imply that $T$ has a nontrivial hyperinvariant subspace.

The following proposition provides the concrete structure of a compact class A operator.

Proposition 3.10. Let $T \in \mathcal{L}(\mathcal{H})$ be a class $A$ operator. If $T$ is compact, then $T=B \oplus C \oplus(-C)$ where $B$ and $C$ are normal. 
Proof. If $T \in \mathcal{L}(\mathcal{H})$ is a class A operator, then $T^{2}$ is $w$-hyponormal from [13. Since $T^{2}$ is compact, it is normal by [3]. Hence $T$ is a square root of a normal operator, and so by [26] we get the following form:

$$
T=B \oplus\left(\begin{array}{cc}
C & D \\
0 & -C
\end{array}\right)
$$

where $B$ and $C$ are normal and $D$ is a positive one-to-one operator commuting with $C$. Since $T$ is also normal by [14], $D$ must be 0 , completing the proof.

If $T \in \mathcal{L}(\mathcal{H})$ and $x \in \mathcal{H}$, then $\left\{T^{n} x\right\}_{n=0}^{\infty}$ is called the orbit of $x$ under $T$, and is denoted by $\mathcal{O}(x, T)$. If $\mathcal{O}(x, T)$ is dense in $\mathcal{H}$, then $x$ is called a hypercyclic vector for $T$. An operator $T \in \mathcal{L}(\mathcal{H})$ is called hypertransitive if every nonzero vector in $\mathcal{H}$ is hypercyclic for $T$. Denote the set of all nonhypertransitive operators in $\mathcal{L}(\mathcal{H})$ by $(\mathrm{NHT})$. The hypertransitive operator problem is the question whether $(\mathrm{NHT})=\mathcal{L}(\mathcal{H})$. The following theorem shows that every class A operator belongs to (NHT).

TheOREM 3.11. If $T \in \mathcal{L}(\mathcal{H})$ is a class $A$ operator, then it is nonhypertransitive.

Proof. If $T$ is not a quasiaffinity, then $\sigma_{\mathrm{p}}(T) \cup \sigma_{\mathrm{p}}\left(T^{*}\right) \neq \emptyset$. Hence $T$ has a nontrivial invariant subspace, and so $T \in(\mathrm{NHT})$. On the other hand, suppose that $T$ is a quasiaffinity. Then so is $T^{2}$. Since $T^{2}$ is $w$-hyponormal from [13], $\widehat{T}^{(2)}$ is hyponormal. Set $S={\widehat{T^{2}}}^{2}$ Since $\widehat{S}={\widehat{T^{2}}}^{(2)}$ is not hypercyclic from [17, there exists a nonzero vector $x \in \mathcal{H}$ such that $\mathcal{O}(x, \widehat{S})$ is not dense in $\mathcal{H}$. Let $S=U|S|$ be the polar decomposition of $S$. Since $U|S|^{1 / 2} \widehat{S}=S U|S|^{1 / 2}$,

$$
S\left(U|S|^{1 / 2} \mathcal{O}(x, \widehat{S})\right)=U|S|^{1 / 2}(\widehat{S} \mathcal{O}(x, \widehat{S})) \subseteq U|S|^{1 / 2} \mathcal{O}(x, \widehat{S}) .
$$

Since $T^{2}$ is a quasiaffinity, so is $S$. Hence $|S|$ is a quasiaffinity and $U$ is unitary. Therefore, $U|S|^{1 / 2} \mathcal{O}(x, \widehat{S})$ is not dense in $\mathcal{H}$. So $S \in(\mathrm{NHT})$. By the same argument as above, we can show that $T^{2} \in(\mathrm{NHT})$. By [4] or [16], $T \in(\mathrm{NHT})$.

COROllary 3.12. If $T \in \mathcal{L}(\mathcal{H})$ is an invertible class $A$ operator, then $T$ and $T^{-1}$ have a common nontrivial invariant closed set.

Proof. This follows from the proof of Theorem 3.11 and [17.

The following theorem, based on the method of [10], gives a necessary and sufficient condition for hypercyclicity of the adjoint of a class A operator.

Theorem 3.13. If $T \in \mathcal{L}(\mathcal{H})$ belongs to class $A$, then $T^{*}$ is hypercyclic if and only if $\sigma_{T}(x) \cap \mathbb{D} \neq \emptyset$ and $\sigma_{T}(x) \cap(\mathbb{C} \backslash \overline{\mathbb{D}}) \neq \emptyset$ for all nonzero $x \in \mathcal{H}$, where $\mathbb{D}=\{z \in \mathbb{C}:|z|<1\}$. 
Proof. Suppose that $T^{*}$ is hypercyclic. Then by Proposition 2.3 of [10, it is enough to show that $\sigma(T)$ meets both $\mathbb{D}$ and $\mathbb{C} \backslash \overline{\mathbb{D}}$. Let $S=\left.T\right|_{\mathcal{M}}$ for some closed $T$-invariant subspace $\mathcal{M}$ and let $x$ be a hypercyclic vector for $T^{*}$. Since $\left(S^{*}\right)^{n} P x=P\left(T^{*}\right)^{n} x$ for each nonnegative integer $n$ where $P$ is the orthogonal projection of $\mathcal{H}$ onto $\mathcal{M}, \overline{\left\{\left(S^{*}\right)^{n}(P x)\right\}_{n=0}^{\infty}}=P\left(\overline{\left\{\left(T^{*}\right)^{n} x\right\}_{n=0}^{\infty}}\right)=$ $P(\mathcal{H})=\mathcal{M}$, i.e., $P x$ is hypercyclic for $S^{*}$. Since $S$ belongs to class A and $S^{*}$ is hypercyclic, $r(S)=\|S\|=\left\|S^{*}\right\|>1$ as mentioned in [23]. Hence, we have $\sigma(T) \cap(\mathbb{C} \backslash \overline{\mathbb{D}}) \neq \emptyset$. On the other hand, in order to prove $\sigma(S) \cap \mathbb{D} \neq \emptyset$, assume that $\sigma(S) \subset \mathbb{C} \backslash \mathbb{D}$. Since $S^{-1}$ is a class A operator by [11 and $\sigma\left(S^{-1}\right) \subset \overline{\mathbb{D}}$, it follows that $\left\|S^{-1}\right\|=r\left(S^{-1}\right) \leq 1$. Since $S^{*}$ is hypercyclic and invertible, $\left(S^{*}\right)^{-1}$ is hypercyclic by [23], and so $\left\|S^{-1}\right\|=\left\|\left(S^{*}\right)^{-1}\right\|>1$ by [23], which is a contradiction. Therefore, $\sigma(S) \cap \mathbb{D} \neq \emptyset$.

Conversely, suppose that $\sigma_{T}(x) \cap \mathbb{D} \neq \emptyset$ and $\sigma_{T}(x) \cap(\mathbb{C} \backslash \overline{\mathbb{D}}) \neq \emptyset$ for all nonzero $x \in \mathcal{H}$. Then we get $\mathcal{H}_{T}(\mathbb{C} \backslash \mathbb{D})=(0)$ and $\mathcal{H}_{T}(\overline{\mathbb{D}})=(0)$. Since $T$ has the property $(\beta)$ by Corollary $3.4, T^{*}$ has the property $(\delta)$. Thus, by Proposition 2.5.14 in $\left[20\right.$, we infer that both $\mathcal{H}_{T^{*}}(\mathbb{D})$ and $\mathcal{H}_{T^{*}}(\mathbb{C} \backslash \overline{\mathbb{D}})$ are dense in $\mathcal{H}$. By using Theorem 3.2 in [10], $T^{*}$ is hypercyclic.

Acknowledgements. This work was supported by Basic Science Research Program through the National Research Foundation of Korea (NRF) grant funded by the Korea government (MEST)(2009-0093125).

\section{References}

[1] E. Albrecht and J. Eschmeier, Analytic functional models and local spectral theory, Proc. London Math. Soc. 75 (1997), 323-348.

[2] A. Aluthge, On p-hyponormal operators for $0<p<1$, Integral Equations Operator Theory 13 (1990), 307-315.

[3] A. Aluthge and D. Wang, w-Hyponormal operators, ibid. 36 (2000), 1-10.

[4] S. I. Ansari, Hypercyclic and cyclic vectors, J. Funct. Anal. 128 (1995), 374-383.

[5] C. Berger, Sufficiently high powers of hyponormal operators have rationally invariant subspaces, Integral Equations Operator Theory 1 (1978), 444-447.

[6] P. Bourdon, Orbits of hyponormal operators, Michigan Math. J. 44 (1997), 345-353.

[7] S. Brown, Hyponormal operators with thick spectrum have invariant subspaces, Ann. of Math. 125 (1987), 93-103.

[8] I. Colojoară and C. Foiaş, Theory of Generalized Spectral Operators, Gordon and Breach, New York, 1968.

[9] J. Eschmeier, Invariant subspaces for subscalar operators, Arch. Math. (Basel) 52 (1989), 562-570.

[10] N. S. Fieldman, V. G. Miller, and T. L. Miller, Hypercyclic and supercyclic cohyponormal operators, Acta Sci. Math. (Szeged) 68 (2002), 965-990.

[11] T. Furuta, Invitation to Linear Operators, Taylor and Francis, 2001.

[12] T. Furuta, M. Ito, and T. Yamazaki, A subclass of paranormal operators including class of log-hyponormal and several related classes, Sci. Math. 1 (1998), 389-403. 
[13] M. Ito and T. Yamazaki, Relations between two inequalities $\left(B^{\frac{r}{2}} A^{p} B^{\frac{r}{2}}\right)^{\frac{p}{p+r}} \geq B^{r}$ and $A^{p} \geq\left(A^{\frac{p}{2}} B^{r} A^{\frac{p}{2}}\right)^{\frac{p}{p+r}}$ and their applications, Integral Equations Operator Theory $44(2002), 442-450$.

[14] I. H. Jeon and B. P. Duggal, On operators with an absolute value conditions, J. Korean Math. Soc. 41 (2004), 617-627.

[15] I. B. Jung, E. Ko, and C. Pearcy, Aluthge transforms of operators, Integral Equations Operator Theory 37 (2000), 437-448.

[16] -, - - - Some nonhypertransitive operators, Pacific J. Math. 220 (2005), 329-340.

[17] C. Kitai, Invariant closed sets for linear operators, Ph.D. Thesis, Univ. of Toronto, 1982.

[18] E. Ko, $k$ th roots of p-hyponormal operators are subscalar operators of order $4 k$, Integral Equations Operator Theory 59 (2007), 173-187.

[19] R. Lange and S. Wang, New Approaches in Spectral Decomposition, Contemp. Math. 128, Amer. Math. Soc., 1992.

[20] K. B. Laursen and M. M. Neumann, An Introduction to Local Spectral Theory, London Math. Soc. Monogr. (N.S.) 20, Clarendon Press, Oxford, 2000.

[21] M. Martin and M. Putinar, Lectures on Hyponormal Operators, Oper. Theory Adv. Appl. 39, Birkhäuser, Basel, 1989.

[22] V. Matache, Operator equations and invariant subspaces, Matematiche (Catania) 49 (1994), 143-147.

[23] V. G. Miller, Remarks on finitely hypercyclic and finitely supercyclic operators, Integral Equations Operator Theory 29 (1997), 110-115.

[24] M. Putinar, Hyponormal operators are subscalar, J. Operator Theory 12 (1984), 385-395.

[25] -, Quasisimilarity of tuples with Bishop's property $(\beta)$, Integral Equations Operator Theory 15 (1992), 1047-1052.

[26] H. Radjavi and P. Rosenthal, On roots of normal operators, J. Math. Anal. Appl. 34 (1971), 653-664.

[27] A. Uchiyama, Weyl's theorem for class A operators, Math. Inequal. Appl. 4 (2001), 143-150.

[28] D. Wang and J. K. Lee, Spectral properties of class A operators, Trends Math. 6 (2003), 93-98.

Sungeun Jung, Eungil Ko, Mee-Jung Lee

Department of Mathematics

Ewha Women's University

120-750 Seoul, Korea

E-mail: ssung105@ewhain.net

eiko@ewha.ac.kr

meejung@ewhain.net

Received September 22, 2009

Revised version January 18, 2010 